\title{
GLOBAL PARAMETRIZATION AND COMPUTATION OF RESONANCE SURFACES FOR PERIODICALLY FORCED OSCILLATORS
}

\author{
BRUCE B. PECKHAM *
}

1998

\begin{abstract}
Periodically forced planar oscillators are typically studied by varying the two parameters of forcing amplitude and forcing frequency. Such differential equations can be reduced via stroboscopic sampling to a two-parameter family of diffeomorphisms of the plane. A bifurcation analysis of this family almost always includes a study of the birth and death of periodic orbits. For low forcing amplitudes, this leads to a now-classic picture of Arnold resonance tongues. Studying these resonance tongues for higher forcing amplitudes requires numerical continuation. Previous work has revealed the usefulness of considering these tongues as projections of surfaces of periodic points from the cartesian product of the phase and parameter planes to the parameter plane. Many surfaces were displayed and described in [MP 1994], but their parametrization and computation was not discussed. In this paper, we do discuss their parametrization and computation. Especially useful are global parametrizations which allow automatic computation of the surfaces. We argue that parametrization by " $f_{\mu}(\mathbf{x})-\mathbf{x}$ " is both more likely to be a global parametrization and more "dynamically natural" than two more obvious parametrizations. As a side benefit, $f_{\mu}(\mathbf{x})-\mathbf{x}$ parametrization leads to a computable way of establishing the nonorientablility of period-two surfaces.
\end{abstract}

Key words. Bifurcation, resonance, periodically forced oscillators, Arnold tongues, numerical continuation.

AMS(MOS) subject classifications. Primary 58F14, 34C15, 70K28.

1. Introduction: Resonance surfaces for forced oscillators. In this introduction we briefly review basic results for two-parameter families of maps of the plane which are generated by periodically forced planar oscillators. The main objects of interest in this paper are resonance surfaces - surfaces of periodic points in the Cartesian product of the phase and parameter planes. The surfaces' projections to the parameter plane are called resonance regions - regions of parameter values for which the corresponding maps have periodic orbits of a certain period. An illustration of three such resonance surfaces for an example described later in the paper is given in Fig. 1a. Their projections to the parameter plane reveal the three correponding resonance regions in Fig. 1b. The fixed-point Hopf bifurcation curve is included in both Figs. 1a and $1 \mathrm{~b}$ for reference. It turns out that resonance surfaces are two-dimenensional manifolds, most often topological disks or mobius strips. We focus on some global parametrizations of these surfaces. The global parametrizations make their computation more automatic and lead to an enhanced understanding of periodic point

*Department of Mathematics and Statistics, University of Minnesota, Duluth, MN 55812 , bpeckham@d.umn.edu. The work was supported in part by NSF grant DMS9505051. 
bifurcations and thus to a better understanding of the dynamics of forced oscillator systems. The exposition and notation follows that of [MP 1994]. More details can be found in that reference and references therein. See especially [AMKA 1986], [P 1988], [P 1990], [MP 1995].

1.1. The periodically forced oscillator setting. A standard general periodically forced oscillator model is given by the equation

$$
\dot{\mathbf{x}}=\mathbf{V}(\mathbf{x})+\alpha \mathbf{W}(\mathbf{x}, \omega t)
$$

where $\mathbf{x} \in \mathbf{R}^{\mathbf{2}}$. The real parameter $\alpha$ is the forcing amplitude, while the real parameter $\omega$ is the forcing frequency. The function $\mathbf{W}$ is periodic with period one in its second variable. We assume that $\mathbf{V}$ and $\mathbf{W}$ are both $C^{\infty}$. When $\alpha=0$, equation (1.1) represents an autonomous vector field. This vector field is assumed to have a repelling equilibrium point $\mathbf{c}_{0}$ inside an attracting periodic orbit $C_{0}$ with frequency $\omega_{0}>0$, and hence period $1 / \omega_{0}$. The periodic orbit is the unforced oscillator; $\omega_{0}$ is its natural frequency.

It is convenient to replace the forcing frequency $\omega$ with the ratio of the natural frequency to the forcing frequency: $\beta \equiv \omega_{0} / \omega$. Restricting $\alpha$ to be nonnegative leaves us with the following parameter space:

$$
\mu \equiv(\beta, \alpha) \in P \equiv(0, \infty) \times[0, \infty)
$$

The stroboscopic family is obtained by following the solutions to equation (1.1), denoted $\phi_{(\beta, \alpha)}(\mathbf{x}, t)$, for time $1 / \omega$, which is the period of forcing. Thus the stroboscopic maps of the plane are defined by

$$
\mathbf{f}_{(\beta, \alpha)}(\mathbf{x}) \equiv \phi_{(\beta, \alpha)}(\mathbf{x}, 1 / \omega)=\phi_{(\beta, \alpha)}\left(\mathbf{x}, \beta / \omega_{0}\right)
$$

This is the two-parameter family of $C^{\infty}$ diffeomorphisms of the plane which we study.

An important observation is that for $\alpha=0$, equation (1.2) defines maps having the unforced oscillator orbit $C_{0}$ as an invariant circle. Further, restricted to $C_{0}$, the maps are rigid rotations with rotation number equal to $\beta$. For small $\alpha$, an invariant circle near $C_{0}$ persists. This implies that for $\alpha$ small, the maps of equation (1.2) can be studied via circle map theory. This is exactly the setting - perturbations of rigid rotations - of the familiar Arnold circle maps family: $x \rightarrow x+\beta+\alpha \sin (x)$. As the forcing amplitude continues to increase, however, the invariant circles tend to shrink in size, eventually disappearing, via a Hopf bifurcation, to a point. Although this last sentence is a great oversimplification of the full dynamical picture, it does point out that in order to understand this transition from dominance by the unforced oscillator ( $\alpha$ small) to dominance by the forcing function ( $\alpha$ large), it is necessary to consider the full two-dimensional phase space rather than just one-dimensional circle maps. 
a)
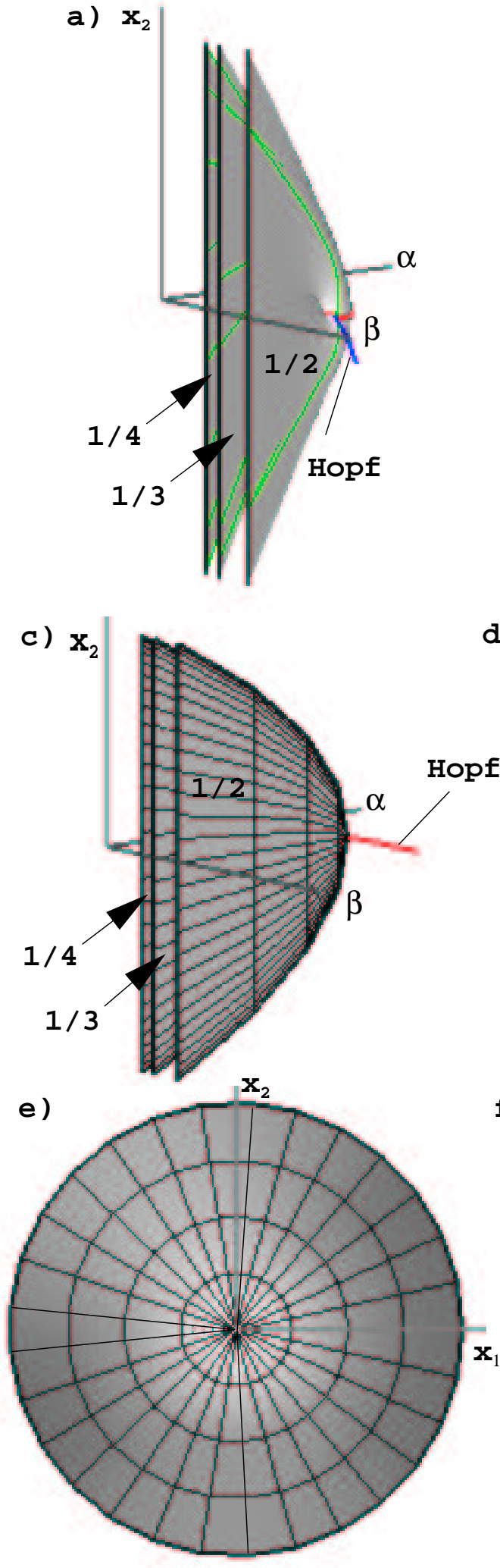

b)

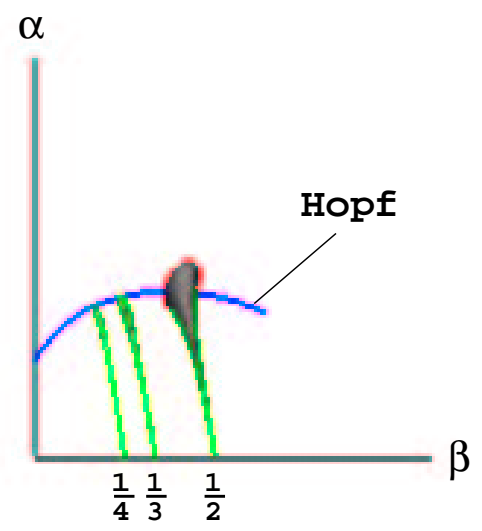

d) $\alpha$

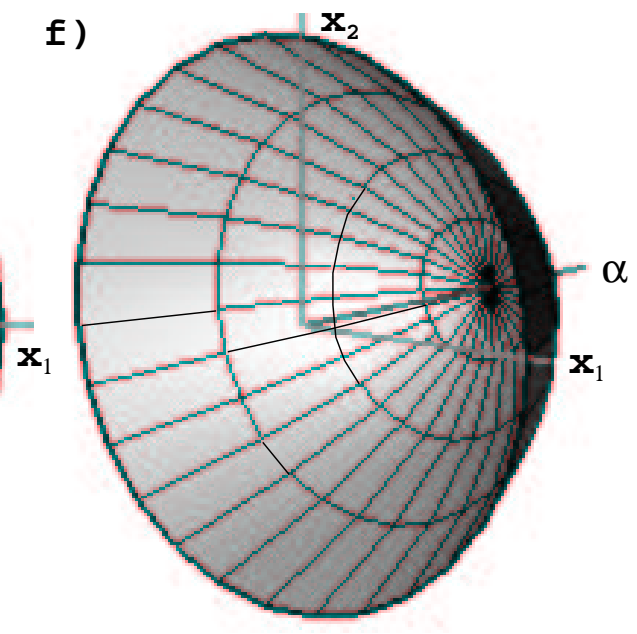

FIG. 1. Resonance surfaces for two periodically forced oscillator (PFO) models. a), b) Generic PFO; c),d),e),f) Nongeneric PFO; e),f) are the same for any $p / q$. 
1.2. Resonance regions. Periodic points of $\mathbf{f}_{(\beta, \alpha)}$ correspond to periodic orbits of equation (1.1) which are said to be in resonance. Thus a resonance region for equation (1.2) is defined to be a region in the parameter plane for which the corresponding maps have a periodic orbit of a certain period. A period- $q$ resonance region, commonly referred to as an Arnold tongue, is known to emanate from each "rational" point $(p / q, 0)$ on the zero forcing amplitude axis. The continuation of these tongues to higher forcing amplitudes is of interest to us in this paper. A more precise definition of resonance region will be given in subsection 1.4 after defining resonance surfaces.

1.3. Resonance surfaces. Resonance regions become more natural objects when they are viewed as projections to the parameter plane of surfaces of periodic points in the cartesian product of the phase and parameter spaces. More specifically, we define a least-period- $q$ surface as

$$
\Gamma(q) \equiv\left\{(\mathbf{x}, \mu) \in P: \mathbf{x} \text { is a least period }-q \text { point for } \mathbf{f}_{\mu}\right\} .
$$

Each $\Gamma(q)$ for $q \geq 2$ typically consists of many distinct components. We are most interested in the components which project to the Arnold tongues in the parameter plane emanating from zero forcing amplitude. See again the surfaces in Fig. 1a and their projections to the parameter plane in Fig. 1b, especially near $\alpha=0$. Note that since the unforced oscillator $C_{0}$ is mapped as a rigid rotation with rotation number $\beta$ by $\mathbf{f}_{(\beta, 0)}$, then $\left\{C_{0} \times(p / q, 0)\right\} \subset \Gamma(q)$ (assuming $p / q$ is reduced).

Thus we define the components of $\Gamma(q)$ which are of interest as

$$
\Gamma_{p / q} \equiv \text { the component of } \Gamma(q) \text { containing }\left\{\mathrm{C}_{0} \times(\mathrm{p} / \mathrm{q}, 0)\right\} .
$$

Previous results have established that these components are distinct for each distinct rational number $p / q$. For example, $\Gamma_{1 / 3}$ and $\Gamma_{2 / 3}$ are necessarily distinct components of $\Gamma(q)$. The proof of this fact involves the definition of a self rotation number for orbits of planar diffeomorphisms which is an extension of the usual rotation number for circle maps [P 1988, $\mathrm{P}$ 1990].

1.4. "The" Resonance Surfaces. The surfaces $\Gamma_{p / q}$ are generically orientable, $C^{\infty}$, and contain a single boundary component: $\left\{C_{0} \times(p / q, 0)\right\}$. This boundary component is really the result of restricting the forcing amplitude to $[0, \infty)$, which in effect cuts each surface off at $\alpha=0$. If we were to allow negative values of $\alpha$, the resonance surfaces would have no boundary components. The $\Gamma_{p / q}$ are typically bounded, but not closed because points on a period- $q$ orbit may coalesce to a periodic orbit of a lower period (which must be a divisor of the original period). This finally leads to the definition of "the" $p / q$ resonance surfaces whose computation we discuss in this paper as:

$$
\text { "The" } p / q \text { resonance surface } \equiv \overline{\Gamma_{p / q}},
$$


where the overbar indicates topological closure. In all examples which we have studied, $\overline{\Gamma_{p / q}}$ is a compact surface for $q \geq 2$. We now precisely define "the" $p / q$ resonance region $A_{p / q}$ as

$$
A_{p / q} \equiv \pi_{\mu}\left(\overline{\Gamma_{p / q}}\right),
$$

where $\pi_{\mu}$ is projection to the parameter plane.

It has been previously shown [P 1988, P 1990] that the closure operation adds only fixed points to $\Gamma_{p / q}$. From a bifurcation viewpoint, these fixed points fall into two classes: $q \geq 3$ and $q=2$. For $q \geq 3$, the fixed points are ( $p / q$ resonant) Hopf bifurcation points - with eigenvalues $e^{ \pm 2 \pi i \frac{p}{q}}$. We denote these points as $H^{p / q}$. Such points are generically isolated in two-parameter families, so their addition to $\Gamma_{p / q}$ via the closure operation leaves $\overline{\Gamma_{p / q}}$ orientable. For $q=2$, the fixed points added to $\Gamma_{p / 2}$ via the closure operation are period-doubling points. They typically form a closed curve in $\overline{\Gamma_{p / 2}}$ and their addition to $\Gamma_{p / 2}$ typically causes $\overline{\Gamma_{p / 2}}$ to be nonorientable, even though $\Gamma_{p / 2}$ is orientable. In subsection 3.4 we discuss further a computational check "around the period doubling curve" which can be done to verify that a specific $\overline{\Gamma_{p / 2}}$ is nonorientable.

Examples of periodically forced oscillators have been constructed $[\mathrm{P}$, in preparation] for which resonance surfaces have topological handles, but no other examples are known to the author. In the case where no handles exist, however, the classification of compact surfaces implies the surfaces must be topological disks for $q \geq 3$, and, assuming nonorientability, mobius strips for $q=2$. The rest of the paper describes different global parametrizations of these surfaces, how the parametrizations are related to the dynamics of the family of stroboscopic maps of eq. (1.2), and how these parametrizations allow easy global computation of the respective surfaces.

2. Global parametrizations of resonance surfaces. In order to better understand global parametrizations of resonance surfaces for a generic family of maps, we first describe how it, and other parametrizations, relate to resonance surfaces for an easily analyzed, but nongeneric example.

2.1. A simple but nongeneric example. We remind the reader that the coarsest description of the dynamics of the maps as a function of the parameters $\mu=(\beta, \alpha)$ is that as $\beta$ increases, the rotation of the corresponding maps increases, and as $\alpha$ increases, the invariant circle shrinks, eventually collapsing to a point. This is a drastic oversimplification, but it helps keep the more detailed dynamical bifurcations in perspective. The following example is constructed to have precisely these two properties. We define the family of maps, $T_{(\beta, \alpha)}(x)$, as the "time-one" map of the following family of planar differential equations, given in polar coordinates:

$$
(\dot{r}, \dot{\theta})=\left((1-\alpha) r-r^{3}, 2 \pi \beta\right) .
$$

This is only a slight variation from the standard model for the Hopf bifurcation for a planar differential equation. In the standard form the 
parameter $\beta$ is a constant (leaving a one-parameter family) and $(1-\alpha)$ is replaced by $\alpha$. For the flow of equation (2.1) the origin undergoes a Hopf bifurcation at $\alpha=1$, as indicated in Figures 1c and 1d. For $\alpha>1$, the origin is a globally attracting equilibrium. For $\alpha<1$, the flow has the origin as a repelling equilibrium, and $C(\sqrt{1-\alpha})$, where $C(a)$ is the circle with center at the origin and radius $a$, as a limit cycle which attracts all other orbits.

The limit cycles of the flow become attracting invariant curves of $T_{(\beta, \alpha)}$. Since the angular coordinate of this time-one map of eq. (2.1) is $\theta \rightarrow \theta+2 \pi \beta$, all $T_{(\beta, \alpha)}$ restricted to the corresponding invariant circles are rigid rotations with rotation number $\beta$. The origin is a fixed point for all parameter values. All periodic orbits except the origin must lie on the invariant curves. Thus, it is relatively easy to see that the least-period$q$ points with rotation number $p / q$ form a surface which is the union of all the invariant circles $(\alpha<1)$ with $\beta=p / q$. If we restrict the surface to $\alpha \geq 0$, as we did in defining $\overline{\Gamma_{p / q}}$ in the general setting above, then $\Gamma_{p / q}=\left\{\left(x_{1}, x_{2}, \beta, \alpha\right):\left(x_{1}, x_{2}\right) \in C(\sqrt{1-\alpha}), \beta=p / q, \alpha \in[0,1)\right\}$. The closure operation adds to $\Gamma_{p / q}$ only the point $(0,0, p / q, 1)$, which is a fixed point. (That is, $(0,0)$ is a fixed point for $T_{(p / q, 1)}$.)

Thus, every $\overline{\Gamma_{p / q}}$ is a topological disk. Its center is the Hopf bifurcation point. Its boundary is the attracting invariant unit circle at zero forcing amplitude: $C(1) \times\{(p / q, 0)\}$. Projections to the $\left(\beta, \alpha, x_{2}\right)$ space of three such $p / q$ resonance surfaces, with $p / q=1 / 4,1 / 3,1 / 2$, are shown in Fig. 1c. Note that in this projection, the invariant circles - four visible on the $1 / 2$ surface, and one each visible on the $1 / 3$ and $1 / 4$ surfaces - collapse to (vertical) line segments. Each $\overline{\Gamma_{p / q}}$ projects to the vertical line segment between $(p / q, 0)$ and $(p / q, 1)$ in the parameter plane. This is illustrated in Fig. 1d for the three indicated surfaces. Each $\overline{\Gamma_{p / q}}$ projects identically to the unit disk in the phase plane (Fig. 1e), and to a paraboloid in the three dimensional $\left(x_{1}, \alpha, x_{2}\right)$ space (Fig. 1f).

The dynamic center. The family of maps, $T_{(\beta, \alpha)}(x)$ induces a map in the four-dimensional phase $\times$ parameter space via

$$
(x,(\beta, \alpha)) \mapsto\left(T_{(\beta, \alpha)}(x),(\beta, \alpha)\right) .
$$

Each resonance surface is invariant under this induced map, which merely rotates the corresponding paraboloid $\overline{\Gamma_{p / q}}$ by $p / q$ of a complete rotation. Thus all points on the $p / q$ surface are period- $q$, except for the origin, which is fixed. From a bifurcation standpoint, this fixed point is a $p / q$ resonant Hopf bifurcation point (although a nongeneric Hopf point for this nongeneric example), referred to as $H^{p / q}$ in subsection 1.4 above. Thus we think of the fixed point as the dynamic center of the surface. For this simple example, the dynamic center, the geometric center, and the center of all three parametrizations defined below coincide, but this will not be the case for the generic example which is presented in subsection 2.2 which follows below. 
Global surface parametrizations. As noted above and illustrated in Fig. 1e, each resonance surface projects homeomorphically to the unit disk in the the phase plane. The inverse of this projection is a global parametrization of that resonance surface. We now formally define this and two additional "projections," whose inverses are each global parametrizations of that resonance surface. In the formulas, $\mathbf{x}=\left(x_{1}, x_{2}\right),|\mathbf{x}|$ is the Euclidean norm of $\mathbf{x}$ in the plane, and $\operatorname{Arg}(\mathbf{x})$ is the angle $(\bmod (2 \pi))$ of $\mathbf{x}$ in the plane.

1. Polar coordinate projection to the phase plane:

$$
\left(x_{1}, x_{2}, \beta, \alpha\right) \in \overline{\Gamma_{p / q}} \mapsto(r, \theta) \equiv(|\mathbf{x}|, \operatorname{Arg}(\mathbf{x})) .
$$

2. Constant $\alpha$ cross sections:

$$
\left(x_{1}, x_{2}, \beta, \alpha\right) \in \overline{\Gamma_{p / q}} \mapsto(\alpha, \phi) \equiv\left(\alpha, \operatorname{Arg}\left(f_{\mu}(x)-x\right)\right) .
$$

3. $f_{\mu}(\mathbf{x})-\mathbf{x}$ :

$$
\left(x_{1}, x_{2}, \beta, \alpha\right) \in \overline{\Gamma_{p / q}} \mapsto(\rho, \phi) \equiv\left(\left|f_{\mu}(x)-x\right|, \operatorname{Arg}\left(f_{\mu}(x)-x\right)\right) .
$$

From this point on in the paper, we will refer to the inverses of these three maps, restricted to the images of the forward maps, as the phase plane, constant $\alpha$, and $f_{\mu}(\mathbf{x})-\mathbf{x}$ parametrizations, respectively, or, equivalently, as the $(r, \theta),(\alpha, \phi)$, and $(\rho, \phi)$ parametrizations, respectively.

Note that the image of the phase plane map is a topological disk, since $r \in[0,1], \theta \in \mathbf{R}$ and we identify $(r, \theta) \sim(r, \theta+2 \pi)$ and $\left(0, \theta_{1}\right) \sim$ $\left(0, \theta_{2}\right)$ for any real $\theta_{1}$ and $\theta_{2}$. This identification is, of course automatic by mapping the polar coordinates to rectangular coordinates: $(r, \theta) \mapsto$ $(r \cos (\theta), r \sin (\theta))$.

Similarly, for the $(\alpha, \phi)$ and $(\rho, \phi)$ parametrizations, we make the identifications: $(\alpha, \phi) \sim(\alpha, \phi+2 \pi)$ and $(\rho, \phi) \sim(\rho, \phi+2 \pi)$, respectively. Analogous to the identification of all angles $\theta$ with $r=0$, we must also make identifications for different angles $\phi$ in the $(\alpha, \phi)$ and $(\rho, \phi)$ parametrizations when $f_{\mu}(x)-x=0$. This identification involves only fixed points on the resonance surfaces. Since we already know the resonance surfaces for this nongeneric model each have a single fixed point (having $\rho=0$ ), and this point is at $\alpha=1$, for the $(\alpha, \phi)$ parametrization we identify $\left(1, \phi_{1}\right) \sim\left(1, \phi_{2}\right)$, and for the $(\rho, \phi)$ parametrization, we identify $\left(0, \phi_{1}\right) \sim\left(0, \phi_{2}\right)$. We will see a much more interesting identification for $\phi$ values with $\rho=0$ when we compute the $1 / 2$ resonance surface for the generic example below.

With the above identifications, we assert that the three maps enumerated above are all injective. The proof of this assertion is mostly contained in the observations made at the beginning of this subsection about the makeup of each $\overline{\Gamma^{p / q}}$. We also note that this claim is corroborated for the phase plane parametrization by Figs 1e and 1f. That the other two are equivalent - for this nongeneric example - to the phase plane parametrization, we note that constant $r$ circles on each $\overline{\Gamma^{p / q}}$ are also constant $\alpha$ circles 
and constant $\rho$ circles. Similarly, constant $\theta$ rays are also constant $\phi$ rays. The relationships between $(r, \theta)$ and $(\rho, \phi)$ are illustrated in Fig. 2 for the specific case $p / q=1 / 4$. In general, a small amount of trigonometry shows that we have $r=\frac{\rho}{2 \cos \left(\frac{q-2 p}{2 q} \pi\right)}, \theta=\phi-\pi \frac{q+2}{2 q}-\frac{p-1}{1} 2 \pi$. As indicated above, $r=\sqrt{1-\alpha}$, or, equivalently, $\alpha=1-r^{2}$. Note that $r$ and $\alpha$ are restricted to $[0,1]$, but $\rho \in\left[0, \rho_{0}\right]$ where $\rho_{0}$ is the distance from $\mathbf{x}$ to $f(\mathbf{x})$ when $\mathbf{x}$ is on the unit circle, and $f(\mathbf{x})$ is obtained from $\mathbf{x}$ by rotating the unit circle by $p / q$ of a complete rotation. In formula: $\rho_{0}=2 \cos \left(\frac{q-2 p}{2 q} \pi\right)$.

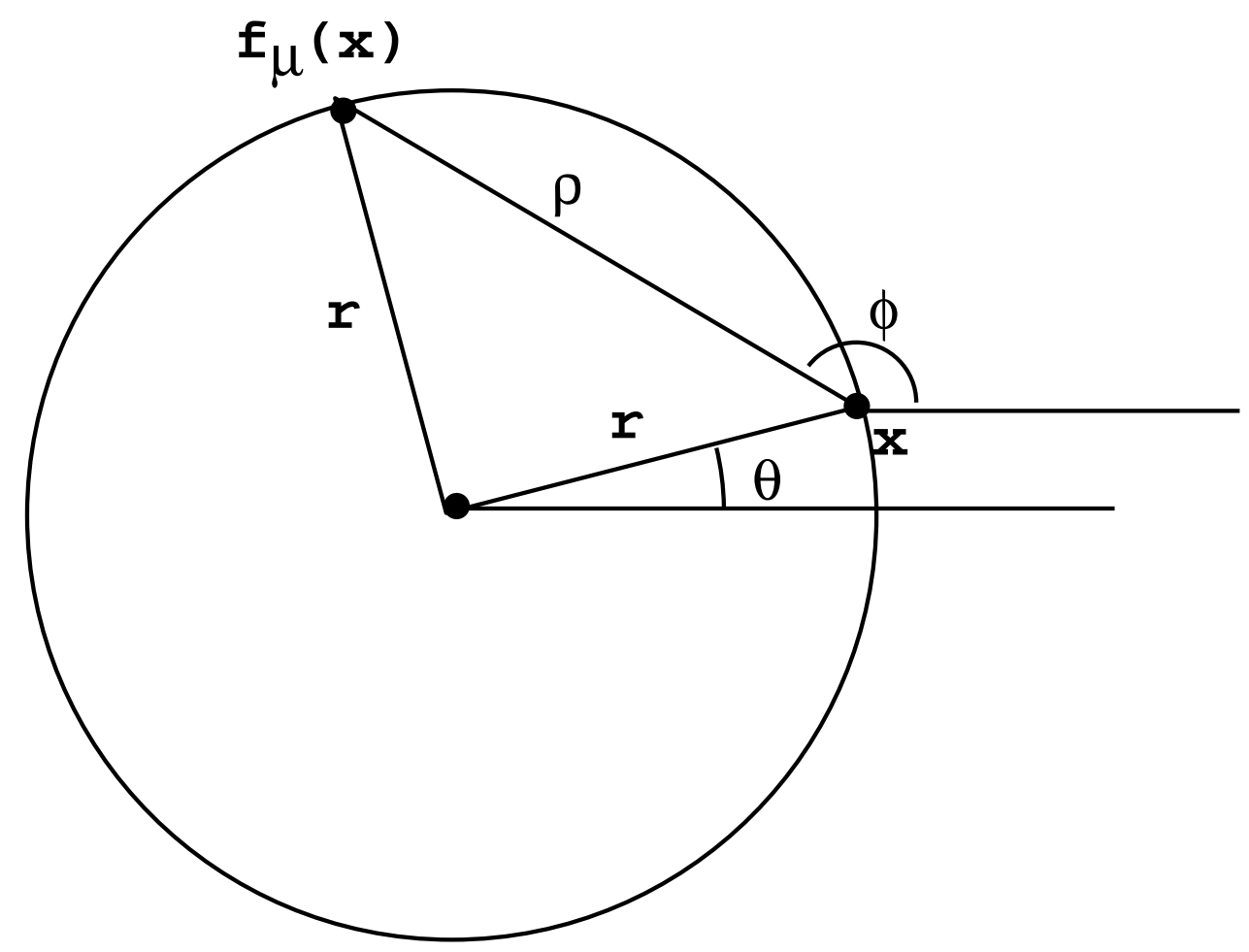

FIG. 2. Relationship between $(r, \theta)$ and $(\rho, \phi)$ for a rigid rotation of a circle by $1 / 4$.

Even better, the model is so simple that we can write down explicitly the inverses of the three projections enumerated above. That is, we can explicitly list the three parametrizations. They are

$$
\begin{gathered}
(\alpha, \phi) \mapsto\left(x_{1}, x_{2}, \beta, \alpha\right)=(\sqrt{1-\alpha} \cos (\phi), \sqrt{1-\alpha} \sin (\phi), p / q, \alpha), \\
(r, \theta) \mapsto\left(x_{1}, x_{2}, \beta, \alpha\right)=\left(r \cos (\theta), r \sin (\theta), p / q, 1-r^{2}\right),
\end{gathered}
$$


and

$$
(\rho, \phi) \mapsto\left(x_{1}, x_{2}, \beta, \alpha\right)=\left(r \cos (\theta), r \sin (\theta), p / q, 1-r^{2}\right),
$$

where $r$ is the multiple of $\rho$ and $\theta$ is the translate of $\phi$ given in the preceding paragraph.

We emphasize the fact that the three parametrizations are essentially the same for this nongeneric example. The only difference is that equal spacing in the $\alpha$ variable is not equal spacing in the other two: $r$ and $\rho$. For example, the circles in Fig. 1e have equal $r$ (and $\rho)$ spacing $(r=$ $1.0, .75, .5, .25$, and $\rho=\sqrt{2} r$ ), but as we can see in Fig. 1c, they have unequal spacing in $\alpha\left(\alpha=1-r^{2}\right)$.

As we shall see below, these parametrizations do not remain the same for generic forced oscillator maps. In fact, not all even remain global parametrizations, even when the surfaces remain topological disks, since the three projection maps enumerated above do not always remain injective. When this happens, there is no inverse map, so the corresponding global parametrization fails.

2.2. A generic caricature example. We investigate the resonance surfaces $\overline{\Gamma_{p / q}}$ for a family of maps that are not constructed exactly as in the introduction, but are constructed with expectations of having generic properties of such families of maps. This family was also studied in [P 1988], [P 1990], [MP 1994] and [MP 1995]. Instead of providing forcing via the nonautonomous term $\mathbf{W}$ in equation (1.1), we provide periodic "impulse" forcing by composing the time-one flow of the autonomous equation $\dot{\mathrm{x}}=$ $\mathbf{V}(\mathbf{x})$ with a map which provides a periodic "kick" to the solution. The kick is defined to be the identity for $\alpha=0$ and increasing in magnitude as $\alpha$ increases. More specifically, this map is defined as

$$
\mathbf{H}_{(\beta, \alpha)} \equiv \mathbf{g}_{\alpha} \circ \mathbf{h}_{\beta},
$$

where $\mathbf{h}_{\beta}(\mathbf{x})$ is the time-one map of the following differential equation, given in polar coordinates:

$$
\dot{r}=\frac{r\left(1-r^{2}\right)}{1+r^{2}}, \dot{\theta}=2 \pi \beta+\frac{1-r^{2}}{1+r^{2}},
$$

and

$$
\mathbf{g}_{\alpha}\left(x_{1}, x_{2}\right)=(1-\alpha)\left(x_{1}-1, x_{2}\right)+(1,0) .
$$

Note that in this setup, $\beta$ is not restricted to be positive, but is allowed to be any real number. So our parameter space is $\mu=(\beta, \alpha) \in P \equiv$ $\mathbf{R} \times[\mathbf{0}, \infty)$.

The $p / q$ resonance surfaces for this caricature map are much too difficult to compute analytically, but they can be computed numerically. We 
have computed three surfaces, for $p / q=1 / 4,1 / 3,1 / 2$. All three were already illustrated in Fig. 1a, projected to the three dimensional $\left(\beta, \alpha, x_{2}\right)$ space. The Hopf bifurcation curve is also included, both for context and for comparison with Fig.'s 1c and 1d. Their projection to the parameter plane is in Fig. 1b. Further views of the individual surfaces are presented in figures $3-7$.

A more detailed discussion of how these surfaces relate to the dynamics and bifurcations of the corresponding family of maps is contained in previous work [P 1988, P 1990, MP 1994, MP 1995]. Here we consider only various parametrizations of the surfaces and the effect the parametrizations have on computing the surfaces. We will see situations in which each of the three parametrizations fails, but that the $(\rho, \phi)$ parametrization is in some sense the best of the three.

The organization of figures $3-5$ are as follows:

- Column 1: Projection to the phase plane, $\left(x_{1}, x_{2}\right)$.

- Column 2: Projection to the $\left(x_{1}, \alpha, x_{2}\right)$ space.

- Column 3: Projection to the $\left(\beta, \alpha, x_{2}\right)$ space.

- Row 1: Full surface.

- Row 2: $(r, \theta)$ parametrization

- Row 3: $(\alpha, \phi)$ parametrization

- Row 4: $(\rho, \phi)$ parametrization

2.2.1. The $1 / 4$ surface. Figure 3 shows views of the $1 / 4$ resonance surface and the eight saddle-node curves that meet at the Hopf bifurcation point $H^{1 / 4}$, which we call the dynamic center of the surface. When projected to the parameter plane, the saddle-nodes become the boundary of the $1 / 4$ resonance region. See Fig. $1 \mathrm{~b}$.

For increased clarity in Fig. 3, the full $10 \times 29$ mesh computed using each of the three parametrizations is not displayed. We display in row 2 only four of the ten constant $r$ curves, equally spaced between $r=1$ and $r=0.1$. The constant $\theta$ curves are connected at $r=0$.

Similarly, the third row displays only four constant $\alpha$ curves, equally spaced between $\alpha=0$ and $\alpha=0.4$. The constant $\phi$ curves are connected at the dynamic center, $H^{1 / 4}$ since the constant $\alpha$ circles degenerate to a point at the $\alpha$ value of $H^{1 / 4}$, which is approximately $\alpha=0.4194396819$.

The fourth row displays only four constant $\rho$ curves, equally spaced between $\rho=\sqrt{2}$ and $\rho=0.1 \sqrt{2}$. The constant $\phi$ curves are connected at the dynamic center, $H^{1 / 4}$ since the constant $\rho$ circles degenerate to a point at $\rho=0$.

In addition, all constant $\theta$ ( $\phi, \phi$, respectively) lines have been removed from the six figures in Columns 2, 3, and rows 2, 3, 4. Saddle-node curves have been left in all figures for reference.

In summary, all three parametrizations are valid global parametrizations for this surface, but the center of the phase space parametrization $(r, \theta)$ does not match the dynamic center, $H^{1 / 4}$, of the surface. 

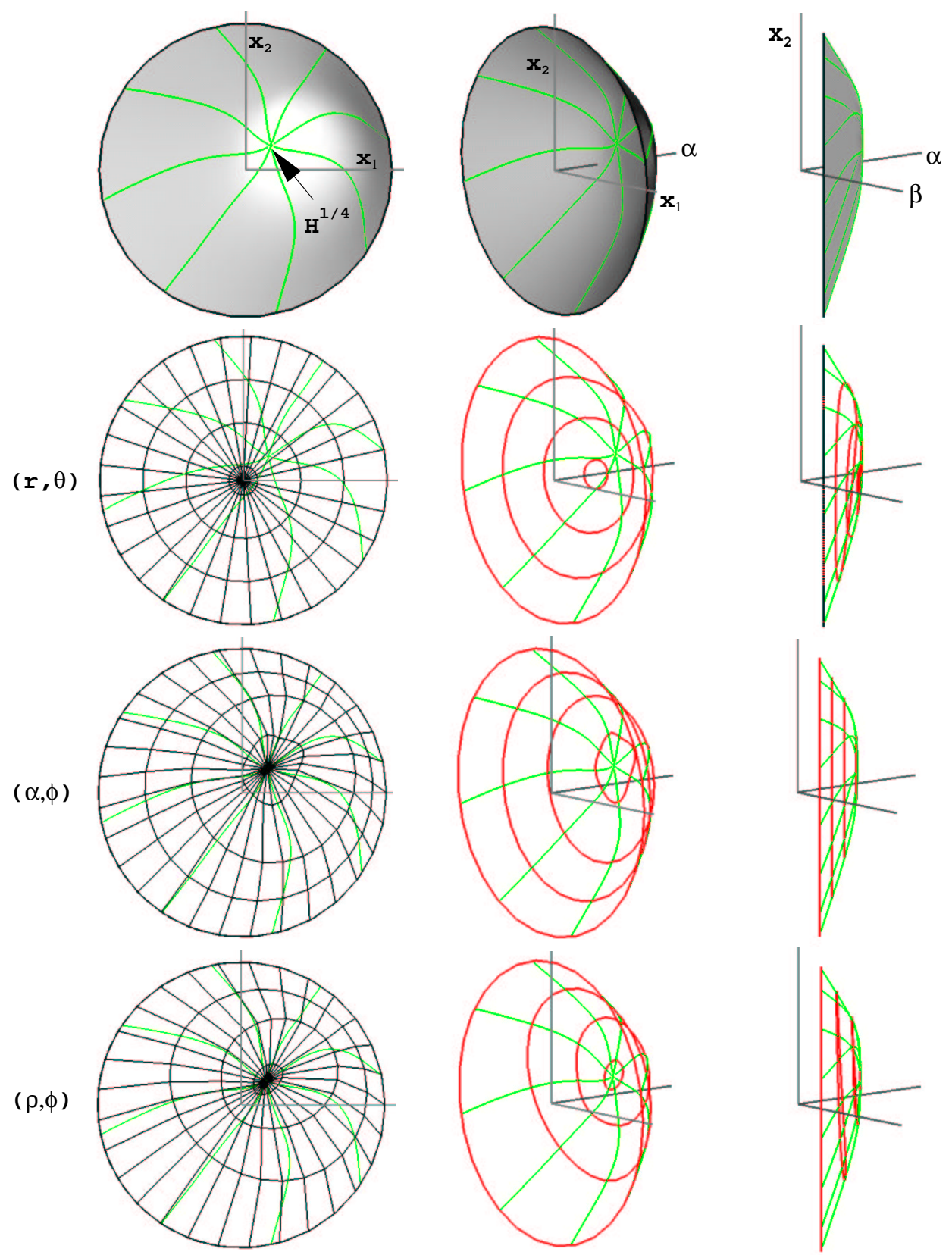

FIG. 3. The $1 / 4$ resonance surface. 
2.2.2. The $1 / 3$ surface. Figure 4 shows views of the $1 / 3$ resonance surface and the three period-three saddle-node curves which "live" on the surface. When projected to the parameter plane, the saddle-nodes become the boundary of the $1 / 3$ resonance region. See Fig. $1 b$ and an enlargement of the top of the $1 / 3$ resonance region in Fig. $7 \mathrm{a}$.

As for the $1 / 4$ surface, we have not displayed the full mesh that was computed for any of rows 2,3 , or 4 . We have displayed in row 2 only four constant $r$ curves between $r=1$ and $r=0.1$, in row 3 only five constant $\alpha$ curves, equally spaced between $\alpha=0$ and $\alpha=0.43$, and in row 4 only four constant $\rho$ curves between $\rho=\sqrt{3}$ and $\rho=0.1 \sqrt{3}$.

Note that in row 3 , column 1 , we have not connected the constant $\phi$ curves to the Hopf point $H^{1 / 3}$. This is because the numerical continuation failed for $\alpha$ values slightly above 0.43. This failure is discussed further below in subsection 2.2.4, and is related to the fact that the saddle-node curves do not meet at the Hopf point $H^{1 / 3}$. It is also consistent with the generic unfolding of a $1 / 3$ resonant Hopf point.

The other two parametrizations, by projection to the phase plane and by $f_{\mu}(\mathbf{x})-\mathbf{x}$, are both valid global parametrizations for the $1 / 3$ surface, but the center of the phase space parametrization $(r, \theta)$ does not match the dynamic center, $H^{1 / 3}$, of the surface.

2.2.3. The $1 / 2$ surface. Because this surface turns out to be a topological mobius strip, there is no way any of the three global parametrizations we have been considering could be valid without alteration. The mobius strip and disk are topologically inequivalent. Thus what is surprising is that the $f_{\mu}(\mathbf{x})-\mathbf{x}$ parametrization "works" rather than that the other two parametrizations fail.

Figure 5 shows views of the $1 / 2$ resonance surface, the two period-two saddle-node curves which "live" on the surface, and the period-doubling circle. From the 1/2 resonance region in Fig. $1 \mathrm{~b}$ and the enlargement of its top portion in Fig. 7c, we see that the boundary of the $1 / 2$ resonance region comprises the saddle-nodes and the "top" part of the period-doubling circle. As for the $1 / 4$ and $1 / 3$ surfaces, we have not displayed the full mesh that was computed for any of rows 2,3 , or 4 . We have displayed in row 2 only three constant $r$ curves, equally spaced between between $r=1$ and $r=0.41$, in row 3 only three constant $\alpha$ curves: $\alpha=0,0.2,0.4$, and in row 4 only six constant $\rho$ curves, equally spaced between $\rho=2$ and $\rho=0.1$.

The $(r, \theta)$ and $(\alpha, \phi)$ parametrizations do not extend to the full $1 / 2$ surface, as is suggested by the "holes" in the center of the meshes displayed in rows 2 and 3 of column 1. These failures are discussed further in subsection 2.2.4 below.

The reason $f_{\mu}(\mathbf{x})-\mathbf{x}$ parametrization can be a global parametrization for both a disk (as it was for the $1 / 4$ and 1/3 surfaces) and a mobius strip, is because the identification at $\rho=0$ is different for the two cases. To become a disk, all points corresponding to $\rho=0$, for any $\phi \in \mathbf{R}$ were identified to a 

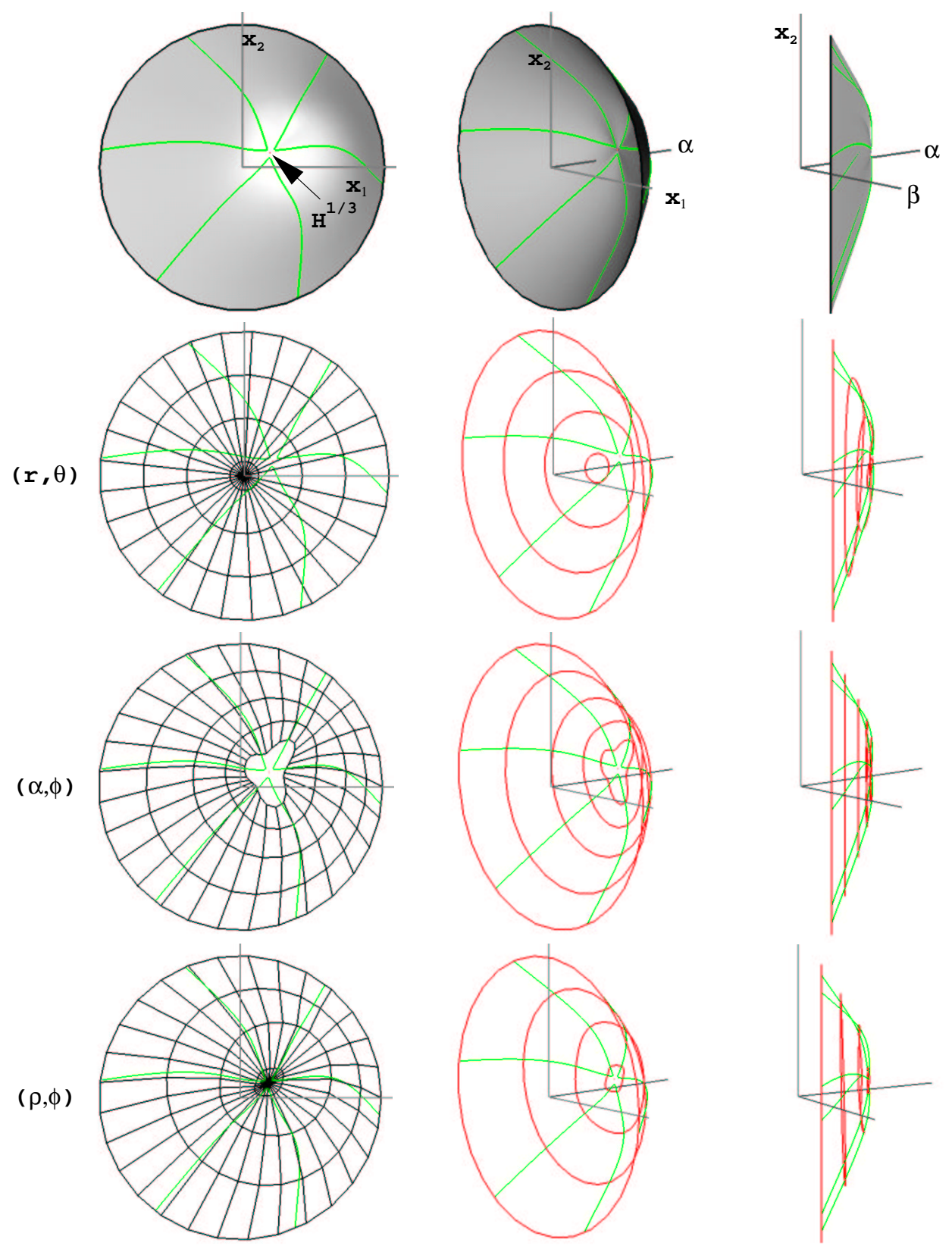

Fig. 4. The $1 / 3$ resonance surface. 

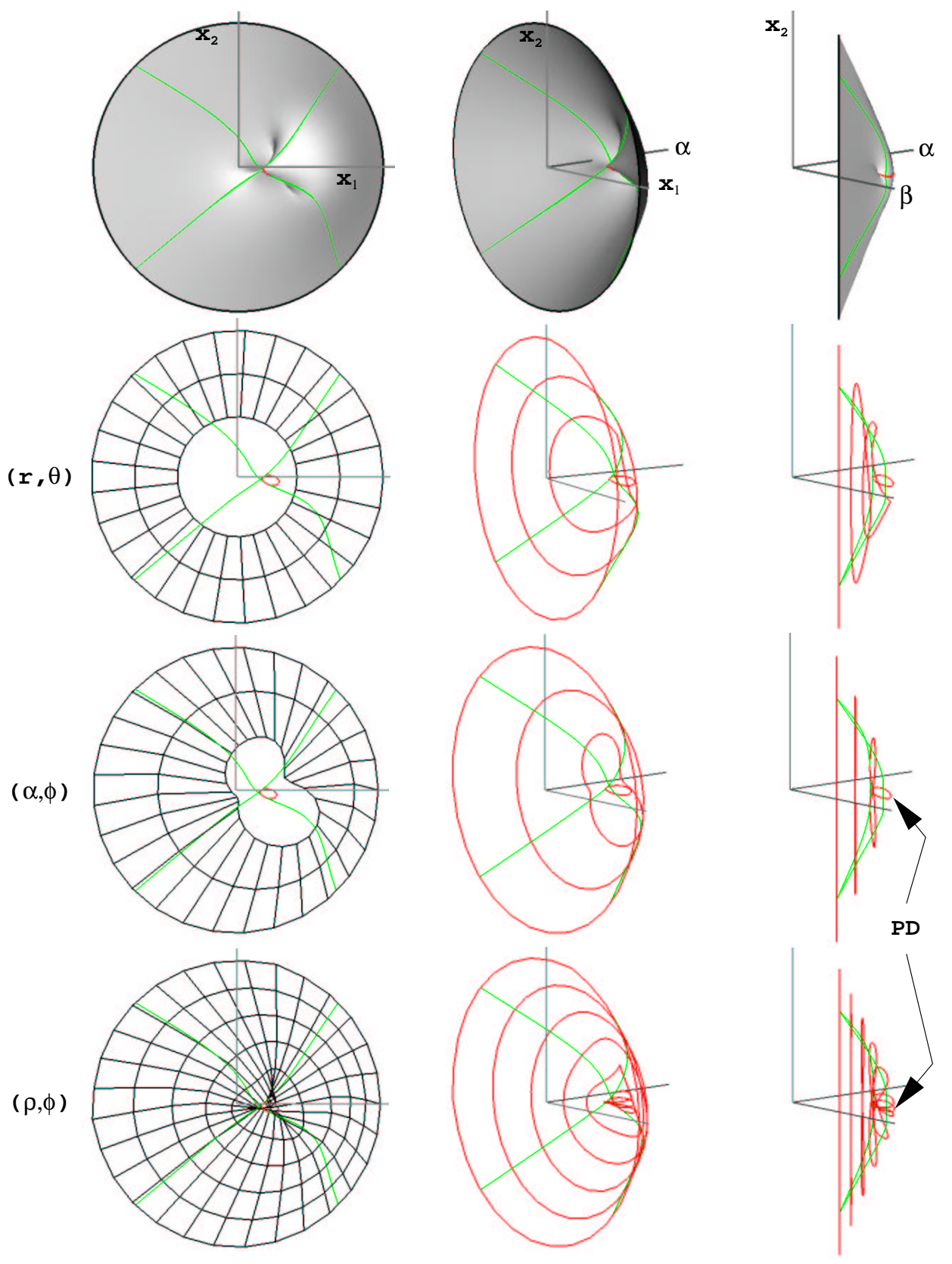

FIG. 5. The $1 / 2$ resonance surface. 
single point. The identification for the mobius strip is instead to a double cover of a circle: $(\rho, \phi)=(0, \phi) \sim(0, \phi+\pi)$. Even better, the circle on which the identification is made $(\rho=0)$ is dynamically significant: it is the period doubling circle. Moreover, sense can be made of the angle $\phi$, even at $\rho=0$ by defining it as the argument of the negative one eigenvector in the phase plane. Note that if $\phi$ is the argument of an eigenvector, then so also is $\phi+\pi$. This is exactly the identification which leaves us with the mobius strip.

Of the Fig. 5 views, the identification at $\rho=0$ is best seen in the row 4 , column 3 figure. The largest constant $\rho$ circle is, as always for the $\left(\beta, \alpha, x_{2}\right)$ projection, collapsed to a vertical line segment. The next five circles appear in this projection as figure-eight curves, the intersection at the center of the figure-eight being an intersection in projection only. The fifth figure eight curve (counting the line segment) has started to fold over slightly at its center. This sixth one has folded over almost completely. Although it appears that this smallest figure eight has three, rather than two, loops, this is because the middle loop (labelled PD) is the perioddoubling circle. Thus, this figure eight is about to collapse onto the period doubling circle, in the process wrapping twice around the period doubling circle.

In order to better visualize how the constant $\rho$ slices limit to the perioddoubling curve as $\rho \rightarrow 0$, we have recomputed just the part of the surface between the sixth figure eight and the period doubling circle referred to in the previous paragraph. This corresponds to $\rho \in[0,0.1]$. The computation of the circle for $\rho=0$ was done separately, and then added to the mesh computed with the $f_{\mu}(\mathbf{x})-\mathbf{x}$ parametrization for $\rho=0.1,0.2 / 3,0.1 / 3$. The parametrization on the period doubling curve was by the argument of the negative one eigenvector. The result is displayed in Fig. 6. This figure corroborates our claim that the negative one eigenvalue parametrization is the limiting parametrization of the $f_{\mu}(\mathbf{x})-\mathbf{x}$ parametrization as $\rho \rightarrow 0$ : the constant $\phi$ rays $d o$ extend smoothly to $\rho=0$ in the figure. Because we have used a $\phi$ mesh size of 29 , which is odd, it is more obvious that constant $\phi$ curves which are $\pi$ apart limit to the same point on the period doubling circle, but from opposite sides. For example, the $\phi=\frac{55}{58} 2 \pi=\frac{13}{29} 2 \pi+\pi$ ray, which would be between the $\phi=\frac{26}{29} 2 \pi$ and $\phi=\frac{27}{29} 2 \pi$ curves in Fig. 6 , would limit on the same point on the period doubling circle $(\rho=0)$ as does the $\phi=\frac{13}{29} 2 \pi$ ray.

A side benefit of understanding this identification at the period-doubling circle is the suggestion of a computational check on whether a given $1 / 2$ surface is orientable. This is discussed further in section 3.4 below.

2.2.4. Failure of global parametrizations. We now describe why the three parametrizations which fail do, in fact, fail.

Constant $\alpha$ for the $1 / 3$ surface. The constant $\alpha$ parametrization almost succeeds for the $1 / 3$ surface, but it will necessarily fail for any 


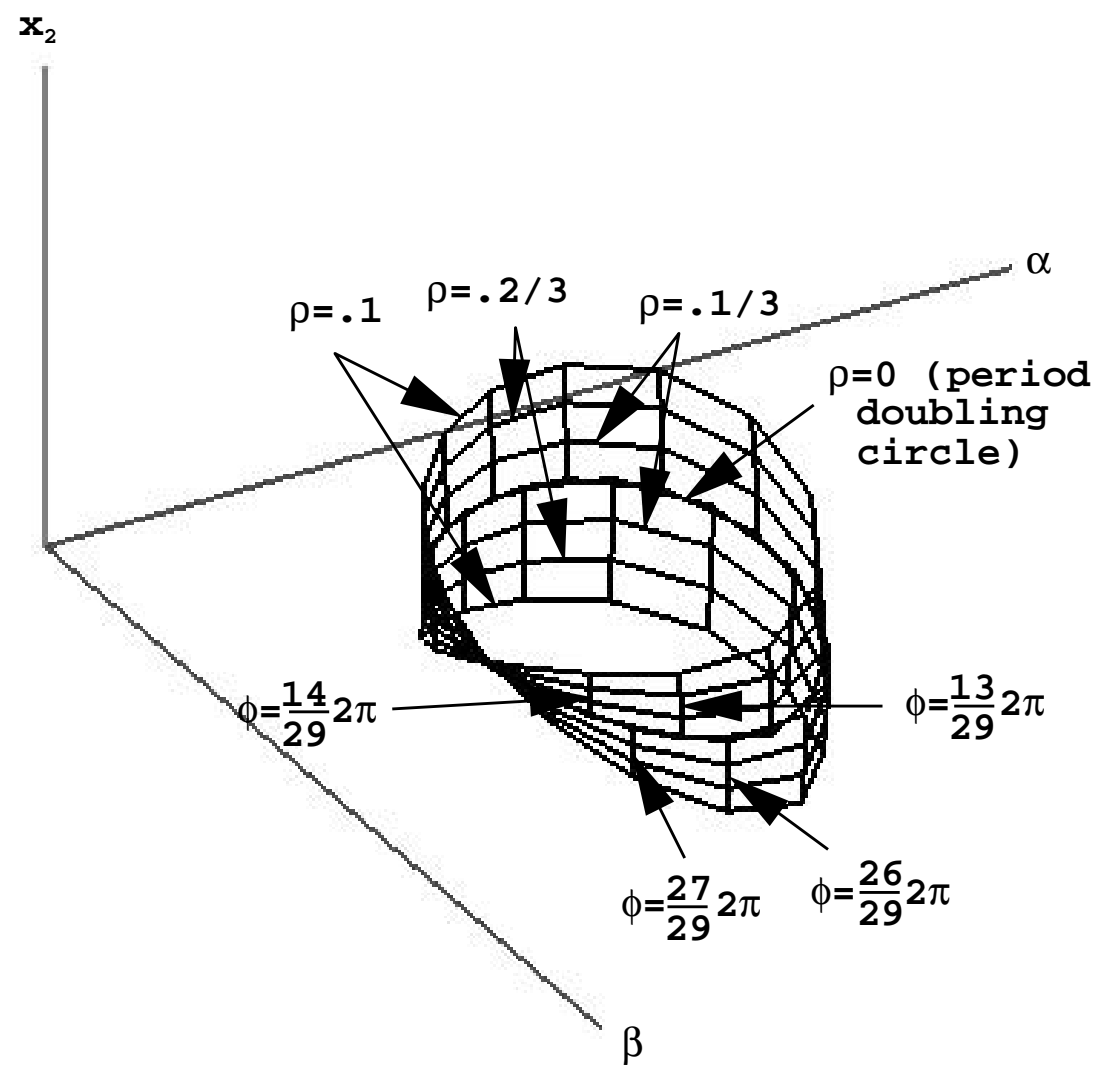

FIG. 6. The $1 / 2$ resonance surface near the period-doubling circle. The angle $\phi$ has been extended to $\phi=0$ as the argument of the negative one eigenvector.

generic period-three surface. This is because period-three resonance regions generically extend above the Hopf bifurcation curve. This leads to the fact that constant $\alpha$ cross sections change from being a single topological circle for $\alpha$ values below the $\alpha$ value of the Hopf point $H^{1 / 3}$, to being three distinct topological circles above $H^{1 / 3}$. This change is illustrated in Figures $7 \mathrm{a}$ and $7 \mathrm{~b}$. Fig. 7a is essentially an enlargement of the top of the $1 / 3$ resonance region of Fig. 1b, near the Hopf curve. The Hopf curve is not shown in Fig. 7a, but the + marks $H^{1 / 3}$, the relevant point on the curve. The two constant $\alpha$ slices and $H^{1 / 3}$ from Fig. 7a are displayed 
projected to the phase plane in Fig. 7b. The outermost constant $\alpha$ curve is for $\alpha=0.43$, which was the innermost $\alpha$ curve in the third row of Fig. 4. The constant $\alpha$ slice for the transition value, the $\alpha$ value of $H^{1 / 3}$, has three petals (not shown) which connect at $H^{1 / 3}$. Note that the $(\alpha, \phi)$ parametrization actually breaks down before reaching the transition value. This is because the angle $\phi$, of $f_{\mu}(\mathbf{x})-\mathbf{x}$, does not vary monotonically as $x$ varies around a constant $\alpha$ circle if $\alpha$ is close enough to (but below) the transition value.

Constant $\alpha$ for the $1 / 2$ surface. Constant $\alpha$ slices of the $1 / 2$ resonance surface are all topological circles in the full four-dimensional phase $\times$ parameter space, except for the two $\alpha$ values for which the constant $\alpha$ curves in the parameter plane are tangent to the period-doubling circle. The slice corresponding to the lower tangency (between $\alpha=0.4$ and 0.43 in Fig. 7c) is a figure eight; the slice corresponding to the upper tangency (above $\alpha=0.5$ in Fig. 7c) is a point. The circles that correspond to $\alpha$ values below the lower tangency, like $\alpha=0.4$, wrap twice around the mobius strip, while the circles which correspond to $\alpha$ values which are between the upper and lower tangency values, like $\alpha=0.43$ and 0.5 , are contractible to a point. These statements are justified in the next paragraph.

Because the projection to the phase plane is not a global parametrization either, we must explain the failure of the constant $\alpha$ parametrization not by looking at how the constant $\alpha$ circles project to the phase plane, but instead, by looking at how the constant $\alpha$ circles project to the "natural" parameter space, $(\rho, \phi)$. This is done in Fig. 7d. With the right and left edges identified as indicated by the arrows, one can see that the $\alpha=0.4$ circle wraps twice around the mobius strip, while the $\alpha=0.43$ and 0.5 circles are contractible to points. It is also easy to visualize on Fig. $7 \mathrm{~d}$ the two critical slices: one between $\alpha=0.4$ and 0.43 which is a figure eight, and the other inside the $\alpha=0.5$ circle which is a single point on the period-doubling curve, $\rho=0$.

Projection to phase plane for the $1 / 2$ surface. This is perhaps easier to visualize that the other two failures. The projection of the surface shown in row 1, column 1 of Fig. 5 is obviously not one-to-one: the surface folds over itself near the center. The innermost constant $r$ curve shown in row 2 , column 1 is near the region where the folds begin. Thus the surface continuation can be expected to fail for lower values of $r$.

2.3. Computation of the surfaces. All numerical continuations performed for this paper were done using the author's continuation software package, To Be Continued [P 1987-present]. Most computations of points on the surfaces for the generic example just described above were done using a globally convergent version of Newton's method in four dimensions. The four variables were the two phase variables $\left(x_{1}, x_{2}\right)$, and the two parameter variables $(\beta, \alpha)$. There were four equations whose simultaneous solution Newton's method sought. Two were determined by the periodic 
a)

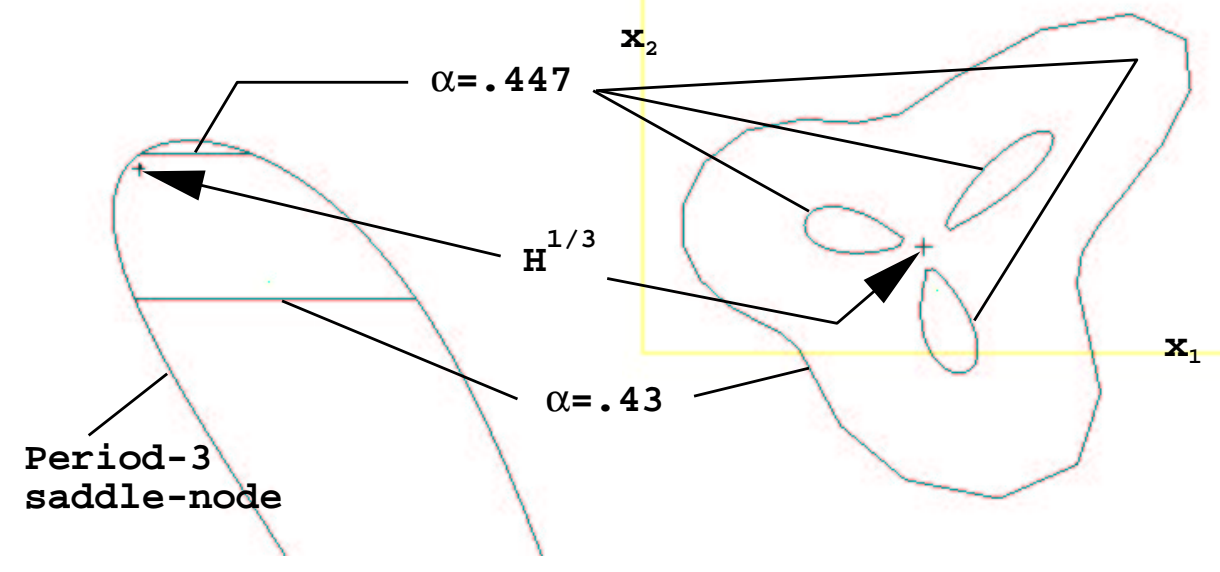

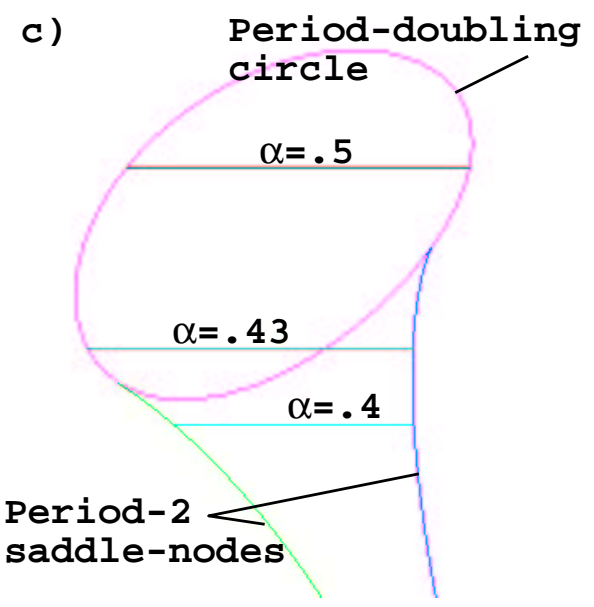

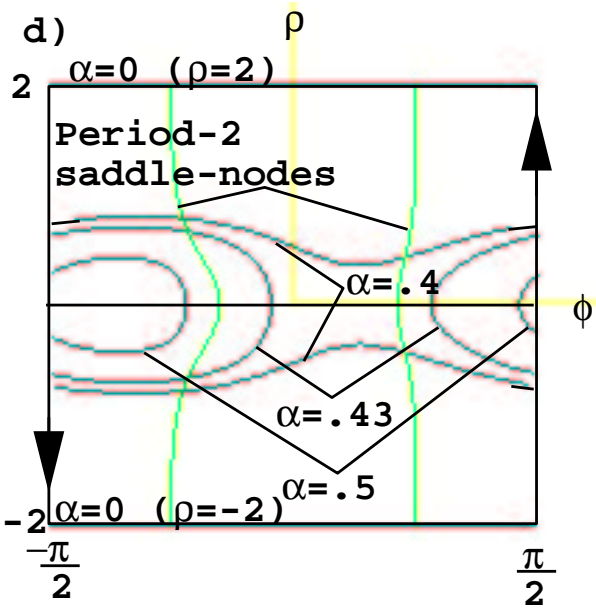

FIG. 7. Failure of the constant $\alpha$ parmetrization. a) $1 / 3$ surface, parameter plane projection: $(\beta, \alpha)$, b) $1 / 3$ surface, phase plane projection: $\left.\left(x_{1}, x_{2}\right), \mathbf{c}\right) 1 / 2$ surface, parameter plane projection: $\left.(\beta, \alpha), \mathbf{d}\right) 1 / 2$ surface, projection to $(\phi, \rho)$ space. Arrows indicate the identification of the right and left edges, $(\pi / 2, \rho) \sim(-\pi / 2, \rho)$, to form a mobius strip. The $\phi$ axis is the period-doubling circle.

point condition $f_{\mu}^{q}(\mathbf{x})-\mathbf{x}=\mathbf{0}$, and the other two were determined by the specific global surface parametrization used (for example, specifying the $(r, \theta)$ values for the phase plane parametrization). An alternative to the $f_{\mu}^{q}(\mathbf{x})-\mathbf{x}=\mathbf{0}$ condition was sometimes used as well. This involved keeping track of the whole periodic orbit instead of just one point on it. It therefore used a higher dimensional version of Newton's method.

The computation using the $f_{\mu}(\mathbf{x})-\mathbf{x}$ parametrization worked auto- 
matically in our generic example for most of the resonance surfaces having periods less than 10. Manual adjustments were sometimes necessary at $\alpha=0$ since the surfaces are more degenerate there (constant $\alpha$ cross sections that project to a line segment in the parameter plane for $\alpha>0$ degenerate to a point at $\alpha=0$.)

\section{Remarks and Conclusions.}

3.1. Implications for other PFO maps. We have compared global parametrizations of three resonance surfaces for a specific caricature of maps obtained from a periodically forced planar oscillator. It is clear especially for the period-two surface, that using the $f_{\mu}(\mathbf{x})-\mathbf{x}$ parametrization is a great advantage. A natural question is whether these same comparisons would hold for other periodically forced oscillator resonance surfaces.

Local theory implies that constant $\alpha$ cross sections will always work for small $\alpha$ on resonance surfaces of all periods greater than or equal to $2 ; f_{\mu}(\mathbf{x})-\mathbf{x}$ will always work near the resonant Hopf bifurcation points, which exist on the surfaces of period 3 or higher. Projection to the phase plane will also work near the resonant Hopf points with the condition that the fixed point not vary "too much" with the system parameters. We believe $f_{\mu}(\mathbf{x})-\mathbf{x}$ will usually work near the period-doubling circle. The other parametrizations have no chance of working near the period-doubling circle.

The question then becomes whether the constant- $\alpha$ parametrization can be extended up to higher values of $\alpha$ to obtain a global parametrization, or whether the other two can be extended down to $\alpha=0$ to obtain a global parametrization. In general, the $f_{\mu}(\mathbf{x})-\mathbf{x}$ parametrization appears to be globally extendible in the greatest number of cases. It requires only that the "size" of the periodic orbit shrinks as $\alpha$ is increased from zero. The phase plane parametrization appears to be extendible only if the "size" of the periodic orbit shrinks as $\alpha$ is increased and the "center of the periodic orbit" doesn't drift too much. The phase plane parametrization, of course, is never useful near the period-doubling circle. The constant- $\alpha$ parametrization appears to work for period- 5 and above (and period- 4 when it is like period5) but never for period 3 and never for $q=2$.

In gereral, the conclusion is that the $f_{\mu}(\mathbf{x})-\mathbf{x}$ parametrization is the most likely to work in most scenarios.

3.2. The superiority of $f_{\mu}(\mathbf{x})-\mathbf{x}$ : two additional scenarios. We have presented a case above for the superiority of the $f_{\mu}(\mathbf{x})-\mathbf{x}$ parametrization over the constant $\alpha$ and projection to phase plane parmetrizations. We consider the three resonance surfaces examined for our generic example to be of the simplest form possible for generic maps generated by periodically forced oscillators. In this subsection, we further support our claim by presenting two further complications, one which causes a constant $\alpha$ parametrization to fail, and the other which causes a projection to 
phase plane parametrization to fail. In both cases, however, the $f_{\mu}(\mathbf{x})-\mathbf{x}$ parametrization remains intact.

The first case is that of a resonance surface for which the Hopf bifurcation is subcritical instead of supercritical. Without describing all the details, the significant result for us is that, near the Hopf bifurcation curve where the resonance region terminates, the Arnold tongue opens up rather than down. The tongue then "turns around" and heads back down toward $\alpha=0$. It is clear that the topology of constant $\alpha$ cross sections changes when $\alpha$ is increased through the $\alpha$ value of the Hopf point $H^{p / q}$. The constant $\alpha$ parametrization thus fails. The projection to phase plane and $f_{\mu}(\mathbf{x})-\mathbf{x}$ parametrizations, however, remain intact.

The second case involves adding an $\alpha$ dependent translation to a family of maps for which the phase plane and $f_{\mu}(\mathbf{x})-\mathbf{x}$ parametrizations both work. Phase plane parametrization will then fail for the new family, but the $f_{\mu}(\mathbf{x})-\mathbf{x}$ parametrization will still work. For example, if we took the maps from our generic example, and translated to the right as $\alpha$ increased, and we translated so much that the Hopf point $H^{1 / 3}$ was completely outside the unit circle, then there would be no way that the $1 / 3$ surface would project in a one-to-one fashion to the phase plane. The constant $\alpha$ and $f_{\mu}(\mathbf{x})-\mathbf{x}$ parametrizations, however, would remain intact. In fact, the $f_{\mu}(\mathbf{x})-\mathbf{x}$ parametrization is unaffected by translations. Its $\rho=0$ points are always the (dynamically significant) fixed points.

3.3. Failure of all three parametrizations. It is possible to construct examples where none of the three parametrizations compared in this paper extend to a global parametrization of a given resonance surface. For example, resonance surfaces can be constructed which have topological handles [P, in preparation].

3.4. Determining the nonorientability of the period-2 surface. In the process of computing the period-two surface using the $f_{\mu}(\mathbf{x})-\mathbf{x}$ parametrization, we noted that the constant $\rho$ circles limit on the perioddoubling circle as $\rho=\left|f_{\mu}(x)-x\right|$ approaches zero. In addition, the $\rho>0$ circles wrap around the period doubling circle twice. It turns out that if one fixes $\phi=\phi_{0}$ and lets $\rho$ approach zero, the limiting point $z_{0}$ on the resonance surface is a period-doubling point, with a negative one eigenvalue. The corresponding eigenvector has angle $\phi$ in the phase plane. Note that the eigenvector could also be represented by the angle $\phi_{0}+\pi$. In fact, if we fix $\phi=\phi_{0}+\pi$ and let $\rho$ approach zero, then the limiting point on the resonance surface is the same point $z_{0}$.

From the unfoldings of period-doubling points, it is clear that the surface of period-two points near a period-doubling point is tangent to the negative one eigenvector. The period-doubling curve itself also lies in the resonance surface $\overline{\Gamma_{p / 2}}$, and this curve is transverse to all the corresponding negative one eigenvectors, since the eigenvectors are confined to the phase plane. Therefore the two vectors: one in the direction tangent to the 
period-doubling curve (oriented, say, by requiring that the projection to the parameter plane is traversed counterclockwise) and the other a negative one eigenvector (with either one of the two of $\phi_{0}$ or $\phi_{0}+\pi$ chosen) form an oriented frame for the surface of period-two points. Let us keep track of this frame as we travel once around the period doubling circle. When we reach the starting point, the vector parallel to the period-doubling circle must be back to where it started. The other vector, however, could either correspond to the same direction $\left(\phi_{0}+2 k \pi\right)$ or the opposite direction $\left(\phi_{0}+\right.$ $\pi+2 k \pi)$. In the former case, the frame has kept its original orientation. In the latter case, the frame has reversed its orientation, indicating that the period-two surface near the period-doubling circle is nonorientable. That is, $\overline{\Gamma_{p / 2}}$ is nonorientable.

This continuation once around a period-doubling circle to see whether orientation is preserved or reversed is easily performed by numerical continuation. Thus we have a quick, computable check on whether a given period-two surface near a period-two circle is nonorientable. Recall that, before adding the period-doubling points to $\Gamma_{p / 2}$ via the closure operation, $\Gamma_{p / 2}$ is orientable. In the case that the period-two surface contains a finite number of topologically circular period-doubling curves (all examples we know of have a single period-doubling curve), we can perform this check on each period-doubling circle. If any one of them indicates nonorientability, the whole surface is nonorientable. If, near each period-doubling circle, the surface is orientable, then the whole surface is orientable.

4. Acknowledgements. Discussions over the years with D. G. Aronson, G. R. Hall, I. G. Kevrekidis, R. P. McGehee, and R. Moeckel on topics related to this paper are gratefully acknowledged.

The author wishes to thank the IMA for support during the Fall 1997 workshop which was instrumental in the development of this paper.

Figures 1 and 3-6 were created from data generated by To Be Continued ... [P 1987-present] and displayed by Geomview [PLM 1993].

\section{REFERENCES}

[AMKA 1986] Aronson D.G., McGehee R.P., Kevrekidis I.G., Aris R., "Entrainment Regions for Periodically Forced Oscillators," Phys. Rev. A 33 (3) 2190-2192, 1986.

[MP 1994] McGehee R.P. and Peckham B.B., "Resonance Surfaces for Forced Oscillators," Geometry Center Research Report GCG70 and Experimental Mathematics, Vol. 3, No. 3, 221-244, 1994.

[MP 1995] McGehee R.P. and Peckham B.B., "Determining the Global Topology of Resonance Surfaces for Periodically Forced Oscillator Families," Proceedings of the Workshop on Normal Forms and Homoclinic Chaos, Fields Institute Communications, AMS, 1995.

[P 1988] Peckham B.B., "The Closing of Resonance Horns for Periodically Forced Oscillators," Ph. D. thesis, University of Minnesota, 1988.

[P 1990] Peckham, B.B., "The Necessity of the Hopf Bifurcation for Periodically Forced oscillators with closed resonance regions," Nonlinearity(3) 261-280, 1990. 
[P 1988-present] Peckham, B.B. To Be Continued ..., a continuation software package for discrete dynamical systems (continually under development).

[P, in preparation] Peckham, B.B., "Handling resonance surfaces."

[PLM 1993] Phillips M., Levy S. and Munzner T., "Geomview: An Interactive Geometry Viewer," Notices of the Amer. Math. Soc. 40 (1993), 985-988. This software and the accompanying manual are available by anonymous ftp from the host geom.umn.edu, in the directory pub/software. 